\title{
Comparing the Effects of Ketorolac and Paracetamol on Postoperative Pain relief after Coronary Artery Bypass Graft surgery. A randomized clinical trial
}

Fatemeh Javaherforoosh Zadeh ( $\nabla$ f_javaherforoosh@yahoo.com )

Ahvaz Jondishapour University of Medical Sciences https://orcid.org/0000-0002-7687-5888

Hasan Abdalbeygi

Ahvaz Jondishapour University of Medical Sciences

Farahzad Janatmakan

Ahvaz Jondishapour University of Medical Sciences

Behnam Gholizadeh

Ahvaz Jondishapour University of Medical Sciences

Research article

Keywords: Ketorolac, Paracetamol, Postoperative analgesia, Visual Analog Score, Cardiac surgery

Posted Date: April 22nd, 2020

DOI: https://doi.org/10.21203/rs.2.23142/v2

License: (c) (1) This work is licensed under a Creative Commons Attribution 4.0 International License.

Read Full License

Version of Record: A version of this preprint was published at Journal of Cardiothoracic Surgery on May 11th, 2020. See the published version at https://doi.org/10.1186/s13019-020-01125-y. 


\section{Abstract}

Introduction : Pain management after coronary artery bypass graft (CABG) surgery remains challenging. Objective This study aimed to compare the effects of Ketorolac and Paracetamol on postoperative CABG pain relief.

Method: This double-blind randomized clinical trial study was conducted in Ahvaz, Iran, from September 2018-December 2019. Two consecutive groups of 60 patients undergoing elective on-pump coronary artery bypass graft surgery. Intervention The patients were divided into $0.5 \mathrm{mg} / \mathrm{kg}$ of ketorolac $\mathrm{mg} / \mathrm{dl}$ and $10 \mathrm{mg} / \mathrm{kg}$ of Paracetamol after surgery for pain management . Primary outcomes were: visual analog pain scale (VAS) at the time point immediately after extubation (baseline) and at 6, 12, 24 and 48 hours and the total dose of morphine consumption. Secondary outcomes included the hemodynamic variables, weaning time, chest tube derange, in-hospital mortality and myocardial infarction. Statistical analysis: The data were analyzed using SPSS version 22(SPSS, Chicago, IL). The Mann-Whitney U-test was used to compare demographic data, VAS scores, vital signs, and side effects. Repeated measurements were tested within groups using Friedman's ANOVA and the Wilcoxon rank-sum test. Values were expressed as means \pm standard deviations. Statistical significance was defined as a p-value $<0.05$.

Results : Compared with baseline scores, there were significant declines in VAS scores in both groups throughout the time sequence (P囚 0.05). The statistical VAS score was slightly higher in the Paracetamol group at most time points, except for the time of $6 \mathrm{~h}$. However, at 24 and 48 hours, the VAS score in group Paracetamol was significantly higher than in group Ketorolac. There were no significant differences between groups about hemodynamic variables.

Conclusion: The efficacy of ketorolac is comparable to that of Paracetamol in postoperative CABG pain relief.

Trial registry: IRCT20150216021098N5. Registered at 2019-09-12

\section{Introduction}

Control of pain management after coronary artery bypass graft (CABG) surgery remains challenging. Incompetently controlled postoperative pain can increase catecholamine levels, triggering myocardial ischemia, stroke, and bleeding complications. Limiting patient mobility, poorly managed postoperative pain can increase the risk of deep vein thrombosis and pneumonia, in addition to harmful psychological consequences such as insomnia and demoralization [1,2]. Postoperative analgesia after cardiac surgery most commonly involves the use of intravenous and oral opioids. Intravenous (IV) opioids, such as morphine, are the analgesics commonly used to provide postoperative pain relief after CABG surgery [3, 4]. However, adverse effects, such as drowsiness, respiratory depression, excessive sedation, biliary spasm, depression of gastrointestinal motility, nausea and vomiting, and, particularly in the elderly, confusion caused by opioids may delay patient recovery and rehabilitation [3,5]. To limit these adverse effects without sacrificing adequate pain management, nonsteroidal anti-inflammatory drugs (NSAIDs), 
increasingly are being applied in the postoperative setting. Although NSAIDs have potential side effects (bleeding, gastrointestinal ulceration, renal dysfunction, and post-operative bone healing ), several studies have noted low complication rates associated with their short-term use after CABG when administered to appropriately selected patients [6, 7]

Paracetamol is usually considered to be a frail inhibitor of the synthesis of prostaglandins (PGs). However, the in vivo effects of Paracetamol are alike to those of the discerning cyclooxygenase-2 (COX-2) inhibitors. It is the most commonly suggested pain-relieving for the treatment of acute pain [8]. Its benefit over NSAIDs is its lack of interfering with platelet functions. Moreover, it is safe to administer to patients with a history of peptic ulcers or asthma [9]. Its mechanism of action may involve a central inhibition of COX-2 $[10,11]$, inhibition of nitric oxide generation via a blockade of the N-methyl-D-aspartate (NMDA) receptor, and activation of the descending serotonergic pathway. Paracetamol can cross the blood-brain barrier, producing a central analgesic effect $[12,13]$

Ketorolac has been used for postoperative analgesia in combination with opioids. Several studies have reported that ketorolac is as effective as morphine or meperidine for analgesia after some types of surgical procedures [14]. However, because many studies report significant side effects of ketorolac, including coagulopathy, gastrointestinal problems, and nephrotoxicity there is increasing interest in the use of other classes of non-opioid analgesics $[15,16]$. It remains unknown whether NSAID utilization rates after CABG have changed since the boxed warning was issued, although some groups have reported their continued use to select cardiac surgery patients $[17,18]$. Management of postoperative pain is a major concern for anesthetists in patients undergoing CABG surgery. Due to the fact that the purpose of studies is to find the methods of pain management with the highest efficiency and the least side effects, and also according to the characteristics mentioned for Ketorolac and Paracetamol, the aim of our study was to compare the analgesic effects of Ketorolac and Paracetamol to find the appropriate anesthetic agent for postoperative CABG pain.

Therefore, this study aimed to compare the effects of Ketorolac and Paracetamol on postoperative CABG pain relief.

\section{Material And Method}

\section{Study design}

This double-blind randomized clinical trial study was conducted in Golestan Hospital, Ahvaz, Iran, from September 2018- December 2019 with Ethics code: IR.AJUMS.REC.1398.050 from Anesthesiology and Pain Research Center, Ahvaz Jundishapur University of Medical Sciences, Ahvaz, Iran, and Trial registration number: IRCT20150216021098N5.

Sixty patients undergoing elective on-pump coronary artery bypass graft surgery. After clearly explaining the objective and potential risks and benefits of the study, a written consent form for participation in the study was obtained from all patients. 
Setting and Patients: Inclusion criteria: Aged 30-70 years, ASA III, Both of sex, Ejection Fraction $\geq 30 \%$, undergoing elective CABG.

Exclusion criteria included: Severe hepatic and renal disease, consumption of anti-inflammatory drugs or antipyretic drugs before the study, redo surgery, history of cerebrovascular accident (CVA), and thrombocytopenia

Randomization: randomization was performed using computer-generated random digits to ensure that patients and investigators were blind to the treatment assignment before study entry, and the allocation was done 1:1 to receive either ketorolac or Paracetamol. Randomization was not performed until electronically confirming the eligibility criteria in the web-based case report form. Randomization was performed centrally without stratification. The sequence was generated by an independent statistician using a random number generator with a 1:1 allocation using random block sizes of 2 . In this study patients and researchers were blinded.

Sample size: The sample size of this study was calculated using the sample size estimation formula. The $95 \%$ confidence interval $(\mathrm{Cl})$ level was considered. The study population consisted of 90 patients. Based on the previous data [19]. After initial screening, 85 patients agreed to participate and provided informed consent. Among them, 25 patients did not have inclusion criteria (Five patients had $\mathrm{EF}<30 \%$, Ten patients had complex surgery, the surgical procedure of Five patients turned into off-pump, Five patients were given antipsychotic drugs or history of seizure). Finally, 60 patients were enrolled in the study and were assigned into two groups of Paracetamol and ketorolac, 30 patients each (Figure1).

\section{Anesthesia protocol}

After arrival to the operation room, standard monitoring included five-lead electrocardiography, pulse oximetry and arterial line for continuous blood pressure monitoring and blood gases were inserted. A standard anesthesia technique was used for all patients. The anesthetic drug doses were calculated according to body weight; midazolam at $0.1 \mathrm{mg} / \mathrm{kg}$ was given as IV premedication. Induction of anesthesia was induced fentanyl $15 \mu \mathrm{g} / \mathrm{kg}$, propofol $1 \mathrm{mg} / \mathrm{kg}$, and pancuronium $0.1-0.15 \mathrm{mg} / \mathrm{kg}$ ). Anesthesia was maintained with a continues infusion of propofol $1-1.5 \mathrm{mg} / \mathrm{kg} / \mathrm{h}$, fentanyl $4 \mu \mathrm{g} / \mathrm{kg} / \mathrm{h}$, $0.25 \mathrm{mg} / \mathrm{kg} / \mathrm{h}$ midazolam, and $0.3 \mathrm{mg} / \mathrm{kg} / \mathrm{h}$ cisatracurium until the end of surgery. Isoflurane supplementation was used at the discretion of the attending anesthesiologist. After induction of general anesthesia, a central venous catheter was introduced. For initiation of cardiopulmonary bypass, 350 $\mathrm{u} / \mathrm{kg}$ heparin was injected to all patients. Heparin dosage was attuned based on goal ACT 450-480 second. After the bypass was terminated, protamine $1 \mathrm{mg} / \mathrm{kg}$ was given for the reversal of heparin. Cardiac surgery and postoperative management were standardized.

After surgery, all the patients were admitted to the cardiovascular ICU, with a standard protocol for sedation, analgesia (propofol $0.5 \mathrm{mg} / \mathrm{kg} / \mathrm{h}$ and morphine sulfate $0.1 \mathrm{mg} / \mathrm{kg} / \mathrm{h}$ ), and management of mechanical ventilation (SIMV mode of ventilation). 
Intervention: Immediately after the transfer of patients to ICU, the intervention began. The patients in the ketorolac group were administered at a rate of $0.5 \mathrm{mg} / \mathrm{kg} \mathrm{IV} \mathrm{admixed} \mathrm{with} 100 \mathrm{ml}$ of normal saline each 6 hours for 24 hours. A bolus may be given at the start of therapy if desired. The patients in the Paracetamol group were given $10 \mathrm{mg} / \mathrm{kg}$ IV of Paracetamol (mixed with normal saline to a total volume of $100 \mathrm{ml}$ ) for 30 minutes each 6 hours for 24 hours. (Dose of ketorolac determined based on Howard et.al study. [20]

Patients were extubated according to the following criteria: responsive and cooperative, p02 of 80-100, oxygenation index of p02/FiO2 > 300, and hemodynamic stability without any inotropes.

After extubation, the patients had access to morphine sulfate with a patient-controlled analgesia device (PCA device; Graseby 3300P, Hoyer, Bremen, Germany) using a standardized protocol: bolus dose of $2 \mathrm{mg}$, dose duration of $2 \mathrm{~min}$, lockout interval of $13 \mathrm{~min}$ (15 min effective lockout time), and with no background infusion and no upper dose limit. Before commencing the PCA, the nurses in the PACU were allowed to give morphine sulfate $2 \mathrm{mgIV}$ before extubation for the treatment of pain if required. Morphine consumption was recorded from the PCA device at the end of the 48-h study period. Per protocol, the primary efficacy variable was cumulative morphine consumption (the combined amount administered via the PCA device and given as rescue doses) at the end of the 48-h postoperative period. If pain relief was insufficient (VAS score $>3$ at rest), nurses were allowed to give an extra bolus of morphine $2 \mathrm{mg}$ IV as a rescue analgesic once an hour. Renal function test (serum creatinine) were measured the evening before surgery and 72 hours after surgery. Bleeding after surgery was measured as chest tube drainage. The number of red cell units transfused was recorded.

Primary outcomes were: visual analog pain scale (VAS) at the time point immediately after extubation (baseline) and at 6, 12, 24 and 48 hours and the total dose of morphine consumption. Secondary outcomes included the hemodynamic variables, weaning time, postoperative bleeding, myocardial infarction, CVA, TIA , in-hospital mortality and postoperative serum creatinine.

\section{VAS score assessment}

Pain intensity levels were subjectively measured using a $10 \mathrm{~cm}$ visual analog pain scale (VAS, $0=$ no pain to $10=$ unbearable pain). We assessed VAS and hemodynamic variables (systolic blood pressure, diastolic blood pressure, heart rate and other parameters ) of each regimen immediately after extubation (baseline) and at 6, 12, 24, and 48 hours.

\section{Statistical analysis}

Numerical variables were reported as mean \pm standard deviation (SD). Quantitative and qualitative variables were measured by independent t-test, and ANOVA test respectively. P value $\leq 0.05$ was considered to be statistically significant. All analyses were performed using SPSS for Windows version 22.0 (SPSS Inc., Chicago, IL, USA) 


\section{Results}

During the study period from September 2018-December 2019, 100 patients undergoing elective on-pump CABG surgery were eligible to participate in the trial. 40 patients did not have inclusion criteria Finally, 60 patients were enrolled in the study and were assigned into two groups of ketorolac and Paracetamol, 30 patients each. (Fig.1)

There were no significant differences between the two groups in terms of demographic characteristics including age, male/female ratio, antiplatelet using, Euro Score $\|$ and duration of cross-clamp time ( $P$ > 0.05) (Table 1).

There were significant differences about morphine consumption in two groups at 24 hours $(0.29 \pm 0.41 \mathrm{mg}$ in Paracetamol group versus $1.71 \pm 0.53 \mathrm{mg}$ in ketorolac group $\mathrm{p}=0.027)$ and 48 hours $(0.22 \pm 0.15 \mathrm{mg}$ in Paracetamol versus $2.18 \pm 0.52 \mathrm{mg}$ in ketorolac group $\mathrm{p}=0.007$ ) after extubation (Fig. 2).

Compared with baseline scores, there were significant declines in VAS scores in both groups throughout the time sequence The statistical VAS score was higher in the Paracetamol group at most time points, except for the time of $6 \mathrm{~h}$. However, at 24 and 48 hours, the VAS score in group Paracetamol was significantly higher than in group Ketorolac (P区 0.05) (Fig. 3).

Comparison of the two groups receiving ketorolac and Paracetamol showed that there was no significant difference between the two groups in terms of platelet count, bleeding time, and chest tube derange Table2. Hemodynamic parameters and SPO2 and postoperative bleeding at different times were not different between the two groups. Table 3

Weaning time significantly lower in the Paracetamol group than the ketorolac group $(p=0.003)$. (Table4)

Comparison of the two groups receiving ketorolac and Paracetamol showed that there was no significant difference between the two groups in terms of the hospital mortality rate MI, CVA, TIA, and postoperative serum creatinine $(P>0.05)$ Table 4 . There were no differences between-group in renal function tests.

\section{Discussion}

Control of pain management in the postoperative care setting is of the greatest importance for patients who experienced CABG. Therefore, pharmacological and interventional approaches have been developed for postoperative analgesia. Currently, there is an increase in the mean age of the patients and the number of comorbidities in patients undergoing CABG. Overall, a method of postoperative analgesia that is cost-effective and comfortable for the patient with minimum complication rates and side effects which also shortens the duration of postoperative stay should be chosen. However, postoperative pain managing is often incomplete by the side effects of opioids; especially when used alone in large doses for an extended period, opioids can lead to acute tolerance and, more seriously, respiratory depression and hypotension. For these explanations, multimodal methods that add non-opioid agents to opioid- 
based regimens are promising. This study aimed to compare the effects of Ketorolac and Paracetamol on postoperative pain management. The main finding in the present study was that ketorolac more effective than Paracetamol to manage postoperative pain patients undergoing CABG surgery. Also, it can reduce postoperative additional analgesic requirements in comparison to Paracetamol with no additional adverse effects. This finding was similar to Amini $S$ et al. study(20). Of course, their study was in congenital cardiac patients.

NSAIDs block the synthesis of prostaglandins through the inhibition of COX-1 and COX-2, thus lowering the production of acute inflammatory response mediators. By decreasing the inflammatory response to surgical trauma, NSAIDs reduce peripheral nociception. NSAIDs also appear to have a central analgesic mechanism, possibly through the inhibition of prostaglandin synthesis within the spinal cord. In general, NSAIDs have a low side-effect profile when administered for the short-term purpose of perioperative analgesia after cardiac surgery $[21,22]$.

Ketorolac is effective at reducing pain, and several studies have reported its safety and efficacy in the perioperative period. In many reports, the use of ketorolac as an adjuvant to a PCA opioid resulted in an opioid-sparing effect ranging from $16 \%$ to $33 \%$ [23]. The hypothesis by which ketorolac exerts these possible beneficial effects is proposed to be related to its COX-1 selectivity and minimal inhibition of COX-2 [24]. As previously discussed, the boxed warning for NSAIDs arose from specific data for the COX2 selective NSAID, celecoxib [25] COX-2 inhibitors selectively reduce prostacyclin synthesis with no effect on thromboxane A2. Prostacyclin is a potent inhibitor of platelet aggregation; its selective blockade by COX-2 inhibitors may upset thrombosis homeostasis and cause adverse cardiovascular events.

Ketorolac, on the other hand, potently blocks platelet aggregation through thromboxane A2 inhibition [24, 26]. This may be beneficial in patients with aspirin resistance to prevent CABG graft failure. The period of this antiplatelet effect can be last up to 24 hours after a distinct dose. Additionally, the antiplatelet effects of ketorolac may offset the risk of hemorrhage in postoperative patients who may be hypercoagulable following exactly off-pump CABG surgery [17].

The authors previously reported the results of a randomized trial that found that oral naproxen is effective as an adjunct for the optimization of pain control and lung recovery after CABG, without increasing the risk of postoperative complications.[27] In contrast to naproxen, intravenous ketorolac can be provided earlier in the postoperative period before the resumption of oral intake. Ketorolac provides an analgesic effect similar to that of fentanyl, but with a lower incidence of postoperative nausea and somnolence, and leads to an earlier return of bowel function. (15)With these advantages over opioids, ketorolac administration ultimately may shorten hospital length of stay.

Paracetamol has been studied in many surgical settings such as functional endoscopic sinus surgery, cholecystectomy, hysterectomy, and orthopedic surgeries with variable favorable results $[28,29]$. The direction of acetaminophen via a nasogastric tube or rectally after surgery is insufficient to accomplish an antipyretic plasma concentration $(10 \mathrm{mg} / \mathrm{ml})$; this was probably mainly because of late gastric emptying after anesthesia and surgery $[13,30]$ In a study conducted by Cattabriga et al. , they found that, 
in patients undertaking cardiac surgery, intravenous paracetamol in combination with tramadol delivers effective pain control[31] [32].

Paracetamol has resulted in hypotension in critically ill patients although this effect could be explained as an allergic phenomenon [33]. The remaining prostaglandin inhibitors seem to exert less marked cardiac depressant effect; in fact, the hemodynamic safety of other NSAIDs such as diclofenac and ketorolac used at antipyretic doses and analgesic doses has been reported in several studies [31].

The hemodynamic effects of NSAIDs used for postoperative pain control in patients undergoing major vascular surgery have been reported in a few studies [34,35]. Although exogenous administration of prostaglandins has marked hemodynamic repercussions, exogenous inhibition of prostaglandin synthesis has a little hemodynamic effect. This could reflect a balance between the reduction in synthesis of prostaglandins with vasodilator and vasoconstrictor actions, with a neutral overall effect. However, NSAIDs must be used cautiously in clinical situations in which prostaglandins have been shown to have advantageous therapeutic effects, such as circulatory insufficiency, shock, myocardial ischemia, coronary spasm, and systemic and pulmonary hypertension; also, NSAIDs may antagonize the effect of antihypertensive medication. In the present study, such patients were excluded and therefore no evaluation of hemodynamic stability when the drugs were present was made.

Our study found no association between the use of $0.5 \mathrm{mg} / \mathrm{kg}$ ketorolac and mortality, $\mathrm{Ml}$, or clinically important hemorrhage. These results, however, are limited by unexpected differences in the baseline characteristics of the number of on-pump CABG patients and STS risk scores. On-pump CABG means a patient placed on cardiopulmonary bypass throughout surgery [36]. The STS risk score is intended for all patients who undergo CABG surgery and helps as a prognosticator of postoperative mortality.[37]

\section{Limitations}

This study has several limitations. First; the sample size was small second; this study was singlecentered. We recommended future trials with a large sample size, multi-center and long duration of follow-up.

\section{Conclusion}

In conclusion, ketorolac and Paracetamol may be produced marked postoperative pain relief after cardiac surgery. The analgesic effects of these compounds were not associated with a clinically significant impairment in hemodynamic function and mortality, $\mathrm{Ml}$, or clinically significant bleeding in postoperative CABG patients. So further study with similarly coordinated groups and the larger sample size is necessary to sufficiently determine the cardiovascular risks associated with administration of IV ketorolac.

\section{Abbreviations}

CABG: Coronary Artery Bypass Graft; VAS: Visual Analog pain Scale 
COX-2: Cyclooxygenase-2 inhibitors; NMDA : N-methyl-D-aspartate ; Euro Score II : European System for Cardiac Operative Risk Evaluation; AO : aortic occlusion; MI: myocardial infarction; CVA: Cerebral vascular accident ;TIA: Transient ischemic attack

\section{Declarations}

Funding: Ahvaz Jundishapur University of Medical Sciences

Conflict of interest: The authors report no conflict of interest

Ethics approval: The local Ethics Committee of Ahvaz Jundishapur University of Medical Sciences, Ahvaz, Iran, approved all of the procedures of this study. IR.AJUMS.REC.1398.050, and Trial registration number: IRCT20150216021098N5.

Consent to participate: Written informed consent was collected from the patients

Consent for publication: Authors provide formal written Consent to Publish before publication

Availability of data: All data were retrieved from the institutional database and are available from the corresponding author upon reasonable request

\section{Acknowledgment}

This paper was a part of a thesis with Ethics code IR.AJUMS.REC.1398.050. This study was designed as a placebo-controlled double-blind clinical trial (IRCT20150216021098N5).We sincerely thank the patients who cooperated with us in this project and supported the research team

Author's contribution: Fatemeh Javaherforooshzadeh and Hasan Abdalbeygi collected data and drafted the manuscript. Behnam Gholizadeh provided study materials and patients' information. Farahzad Janatmakan conceived of the study and participated in its design. All authors read and approved the final manuscript

\section{References}

1. Apfelbaum JL, Chen C, Mehta SS, Gan TJJA, Analgesia: Postoperative pain experience: results from a national survey suggest postoperative pain continues to be undermanaged. 2003, 97(2):534-540.

2. Brennan F, Carr DB, Cousins MJA, Analgesia: Pain management: a fundamental human right. 2007, 105(1):205-221.

3. Liu SS, Block BM, Wu CLJATJotASoA: Effects of Perioperative Central Neuraxial Analgesia on Outcome after Coronary Artery Bypass SurgeryA Meta-analysis. 2004, 101(1):153-161. 
4. Bainbridge D, Martin JE, Cheng DCJCJoA: Patient-controlledversus nurse-controlled analgesia after cardiac surgery-a meta-analysis. 2006, 53(5):492.

5. Ucak A, Onan B, Sen H, Selcuk I, Turan A, Yilmaz ATJJoc, anesthesia v: The effects of gabapentin on acute and chronic postoperative pain after coronary artery bypass graft surgery. 2011, 25(5):824829.

6. Hynninen MS, Cheng DC, Hossain I, Carroll J, Aumbhagavan SS, Yue R, Karski JMJCJoA: Nonsteroidal anti-inflammatory drugs in treatment of postoperative pain after cardiac surgery. 2000 , 47(12):1182-1187.

7. Kulik A, Ruel M, Bourke M, Sawyer L, Penning J, Nathan H, Mesana T, Bédard PJEjoc-ts:

Postoperativenaproxen after coronary artery bypass surgery: a double-blind randomizedcontrolledtrial. 2004, 26(4):694-700.

8. Sachs CJJAfp: Oral analgesics for acute nonspecific pain. 2005, 71(5).

9. Hyllested M, Jones S, Pedersen J, Kehlet HJBjoa: Comparative effect of paracetamol, NSAIDs or their combination in postoperative pain management: a qualitative review. 2002, 88(2):199-214.

10. Graham GG, Scott KFJAjot: Mechanism of action of paracetamol. 2005, 12(1):46-55.

11. Remy C, Marret E, Bonnet FJBjoa: Effects of acetaminophen on morphine sideeffects and consumption after major surgery: meta-analysis of randomized controlled trials. 2005, 94(4):505513.

12. Bonnefont J, Courade J, Alloui A, Eschalier AJD: Antinociceptive mechanism of action of paracetamol. 2003, 63:1-4.

13. Bannwarth B, Netter P, Lapicque F, Gillet P, Pere P, Boccard E, Royer R, Gaucher AJBjocp: Plasma and cerebrospinal fluid concentrations of paracetamol after a single intravenous dose of propacetamol. 1992, 34(1):79-81.

14. De Oliveira GS, Agarwal D, Benzon HTJA, Analgesia: Perioperative single dose ketorolac to prevent postoperative pain: a meta-analysis of randomized trials. 2012, 114(2):424-433.

15. Allen C, Hopewell S, Prentice A, Gregory DJCDoSR: Nonsteroidal anti-inflammatory drugs for pain in women with endometriosis. 2009(2).

16. Tabrizian P, Giacca M, Prigoff J, Tran B, Holzner ML, Chin E, Palese M, Herron D, Arvelakis A, Rudow DLJPiT: Renal Safety of Intravenous Ketorolac Use After Donor Nephrectomy. 2019:1526924819855360.

17. Engoren M, Hadaway J, Schwann TA, Habib RHJTAots: Ketorolac improves graft patency after coronary artery bypass grafting: A propensity-matched analysis. 2011, 92(2):603-609.

18. Engoren MC, Habib RH, Zacharias A, Dooner J, Schwann TA, Riordan CJ, Durham SJ, Shah AJJoc, anesthesia v: Postoperative analgesia with ketorolac is associated with decreased mortality after isolated coronary artery bypass graft surgery in patients already receiving aspirin: a propensitymatched study. 2007, 21(6):820-826.

19. Sakpal TV: Sample size estimation in clinical trial. Perspect Clin Res 2010, 1(2):67-69. 
20. Howard ML, Warhurst RD, Sheehan C: Safety of Continuous Infusion Ketorolac in Postoperative Coronary Artery Bypass Graft Surgery Patients. Pharmacy (Basel) 2016, 4(3):22.

21. Rahman MM, Alam MB, Islam MA, Haque AAJJoM: Non steroidal anti inflammatory drugs-an overview. 2006, 7(1):20-31.

22. Jahnavi K, Reddy PP, Vasudha B, Narender BJJoDD, Therapeutics: Non-steroidal anti-inflammatory drugs: an overview. 2019, 9(1-s):442-448.

23. Ready L, Brown C, Stahlgren L, Egan K, Ross B, Wild L, Moodie J, Jones S, Tommeraasen M, Trierwieler MJA: Evaluation of intravenous ketorolac administered by bolus or infusion for treatment of postoperative pain. A double-blind, placebo-controlled, multicenter study. 1994, 80(6):1277-1286.

24. Warner TD, Giuliano F, Vojnovic I, Bukasa A, Mitchell JA, Vane JRJPotNAoS: Nonsteroid drug selectivities for cyclo-oxygenase-1 rather than cyclo-oxygenase-2 are associated with human gastrointestinal toxicity: a full in vitro analysis. 1999, 96(13):7563-7568.

25. Nussmeier NA, Whelton AA, Brown MT, Langford RM, Hoeft A, Parlow JL, Boyce SW, Verburg KMJNEJOM: Complications of the COX-2 inhibitors parecoxib and valdecoxib after cardiac surgery. 2005, 352(11):1081-1091.

26. Funk CD, FitzGerald GAJJocp: COX-2 inhibitors and cardiovascular risk. 2007, 50(5):470-479.

27. Kulik A, Ruel M, Bourke M, Sawyer L, Penning J, Nathan H, Mesana T, Bédard P:

Postoperativenaproxen after coronary artery bypass surgery: a double-blind randomizedcontrolledtrial. European journal of cardio-thoracic surgery 2004, 26(4):694-700.

28. Berger MM, Berger-Gryllaki M, Wiesel PH, Revelly J-P, Hurni M, Cayeux C, Tappy L, Chioléro RJCcm: Intestinal absorption in patients after cardiac surgery. 2000, 28(7):2217-2223.

29. Arslan M, Celep B, Cicek R, Kalender HÜ, Yılmaz HJJorimstojolUoMS: Comparing the efficacy of preemptive intravenous paracetamol on the reducing effect of opioid usage in cholecystectomy. 2013, 18(3):172.

30. Jebaraj B, Maitra S, Baidya DK, Khanna PJPr, treatment: Intravenous paracetamol reduces postoperative opioid consumption after orthopedic surgery: a systematic review of clinical trials. 2013, 2013.

31. Douzjian DJ, Kulik A: Old drug, new route: a systematic review of intravenous acetaminophen after adult cardiac surgery. Journal of cardiothoracic and vascular anesthesia 2017, 31(2):694-701.

32. Cattabriga I, Pacini D, Lamazza G, Talarico F, Di Bartolomeo R, Grillone G, Bacchi-Reggiani LJEJoC-ts: Intravenous paracetamol as adjunctive treatment for postoperative pain after cardiac surgery: a double blind randomized controlled trial. 2007, 32(3):527-531.

33. Jóźwiak-Bebenista M, Nowak JZJApp: Paracetamol: mechanism of action, applications and safety concern. 2014, 71(1):11-23.

34. Maddali MM, Kurian E, Fahr JJJoca: Extubation time, hemodynamic stability, and postoperative pain control in patients undergoing coronary artery bypass surgery: An evaluation of fentanyl, remifentanil, and nonsteroidal antiinflammatory drugs with propofol for perioperative and postoperative management. 2006, 18(8):605-610. 
35. Roediger L, Larbuisson R, Lamy MJEjoA: New approaches and old controversies to postoperative pain control following cardiac surgery. 2006, 23(7):539-550.

36. Møller $\mathrm{CH}$, Steinbrüchel DA: Off-pump versus on-pump coronary artery bypass grafting. Current cardiology reports 2014, 16(3):455.

37. Bhatt DL, Drozda JP, Shahian DM, Chan PS, Fonarow GC, Heidenreich PA, Jacobs JP, Masoudi FA, Peterson ED, Welke KF: ACC/AHA/STS statement on the future of registries and the performance measurement enterprise: a report of the American College of Cardiology/American Heart Association Task Force on Performance Measures and The Society of Thoracic Surgeons. Journal of the American College of Cardiology 2015, 66(20):2230-2245.

\section{Tables}

Table 1. Demographic Data of patients in Paracetamol and ketorolac groups

\begin{tabular}{|c|c|c|c|}
\hline Parameter & $\begin{array}{c}\text { Paracetamol } \\
(\mathbf{n = 3 0 )}\end{array}$ & $\begin{array}{c}\text { Ketorolac group } \\
(\mathbf{n = 3 0})\end{array}$ & $\begin{array}{c}\text { P- } \\
\text { value }\end{array}$ \\
\hline Male, n(\%) & $18(60)$ & $19(63.33)$ & 0.453 \\
\hline Age (year) & $58.41 \pm 1.82$ & $61.83 \pm 1.54$ & 0.159 \\
\hline Weight(kg) & $70.63 \pm 10.10$ & $74.80 \pm 31.14$ & 0.475 \\
\hline Height(cm) & $173.03 \pm 10.16$ & $163.29 \pm 27.20$ & 0.063 \\
\hline Outpatient use of NSAIDs or & $20(67)$ & $21(70)$ & 0.21 \\
\hline antiplatelet, n (\%) & & & 0.489 \\
\hline Euro Score \| (\%), mean \pm SD & $2.63 \pm 2.65$ & $2.86 \pm 2.83$ & 0.09 \\
\hline Duration of AO(min) & $55 \pm 38$ & $49 \pm 30$ & \\
\hline
\end{tabular}

Values are mean \pm sd or number of patients. ; Euro Score $\|:_{\text {_ }}$ European System for Cardiac Operative Risk Evaluation; AO _ aortic occlusion.SD: Standard deviation

Table2. Effect of administration of ketorolac and Paracetamol on Bleeding Time, Platelet and post-operative bleeding 


\begin{tabular}{|c|c|c|c|}
\hline parameter & Paracetamol (n=30) & Ketorolac & p-value \\
& & $(\mathrm{n}=30)$ & \\
\hline BT in $24 \mathrm{hr} .(\mathrm{S})$ & $121.1 \pm 2.73$ & $126.8 \pm 3.41$ & 0.417 \\
\hline BT in $48 \mathrm{hr} .(\mathrm{S})$ & $129.6 \pm 34.56$ & $132.4 \pm 23.87$ & 0.356 \\
\hline Decrease in platelet, median & $74(11-121)$ & $72(19-153)$ & 0.17 \\
\hline Post-operative bleeding(CC) & $285.25 \pm 65.6$ & $302.05 \pm 68.76$ & 0.65 \\
& & & \\
\hline
\end{tabular}

BT: Bleeding Time; S: Second ; CC: milliliter

Table 3. Effect of administration of ketorolac and Paracetamol on hemodynamic variables

\begin{tabular}{|c|c|c|c|}
\hline parameter & Paracetamol $(\mathrm{n}=30)$ & Ketorolac(n=30) & P-value \\
\hline MAP & $87.742(11.026)$ & $86.451(8.958)$ & 0.59 \\
\hline HR & $86.2 \pm 7.2$ & $85.5 \pm 11.7$ & 0.44 \\
\hline SpO2 & $98.4 \pm 1.4$ & $98.4 \pm 1.3$ & 0.8 \\
& & & \\
\hline
\end{tabular}

HR: Heart rate; MAP: Mean arterial blood pressure; SpO2: Arterial oxygen saturation

Table4. Effect of administration of ketorolac and Paracetamol on outcomes 


\begin{tabular}{|l|l|l|l|}
\hline Parameter & Paracetamol (n=30) & Ketorolac(n=30) & P-value \\
\hline WT, (hr.) & $11.76 \pm 1.21$ & $18.56 \pm 1.14$ & $0.003^{\mathrm{a}}$ \\
\hline MI, n (\%) & $0(0)$ & $0(0)$ & - \\
\hline Hospital mortality, n (\%) & $0(0)$ & $0(0)$ & - \\
\hline CVA, n(\%) & $0(0)$ & $0(0)$ & - \\
\hline TIA, n(\%) & $0(0)$ & $0(0)$ & - \\
\hline Postoperative Cr (mg/dl) & $0.99 \pm 0.12$ & $1.01 \pm 0.16$ & 0.81 \\
\hline
\end{tabular}

hr: Hour ;n: number; WT: Weaning time ;MI: myocardial infarction; CVA: Cerebral vascular accident TIA: Transient ischemic attack SD: standard deviation;; Cr: Serum-creatinine; a means significant

\section{Figures}


Excluded $(n=40)$

- Not meeting inclusion criteria $(n=28)$

- Declined to participate $(n=7)$

- Other reasons $(n=5)$

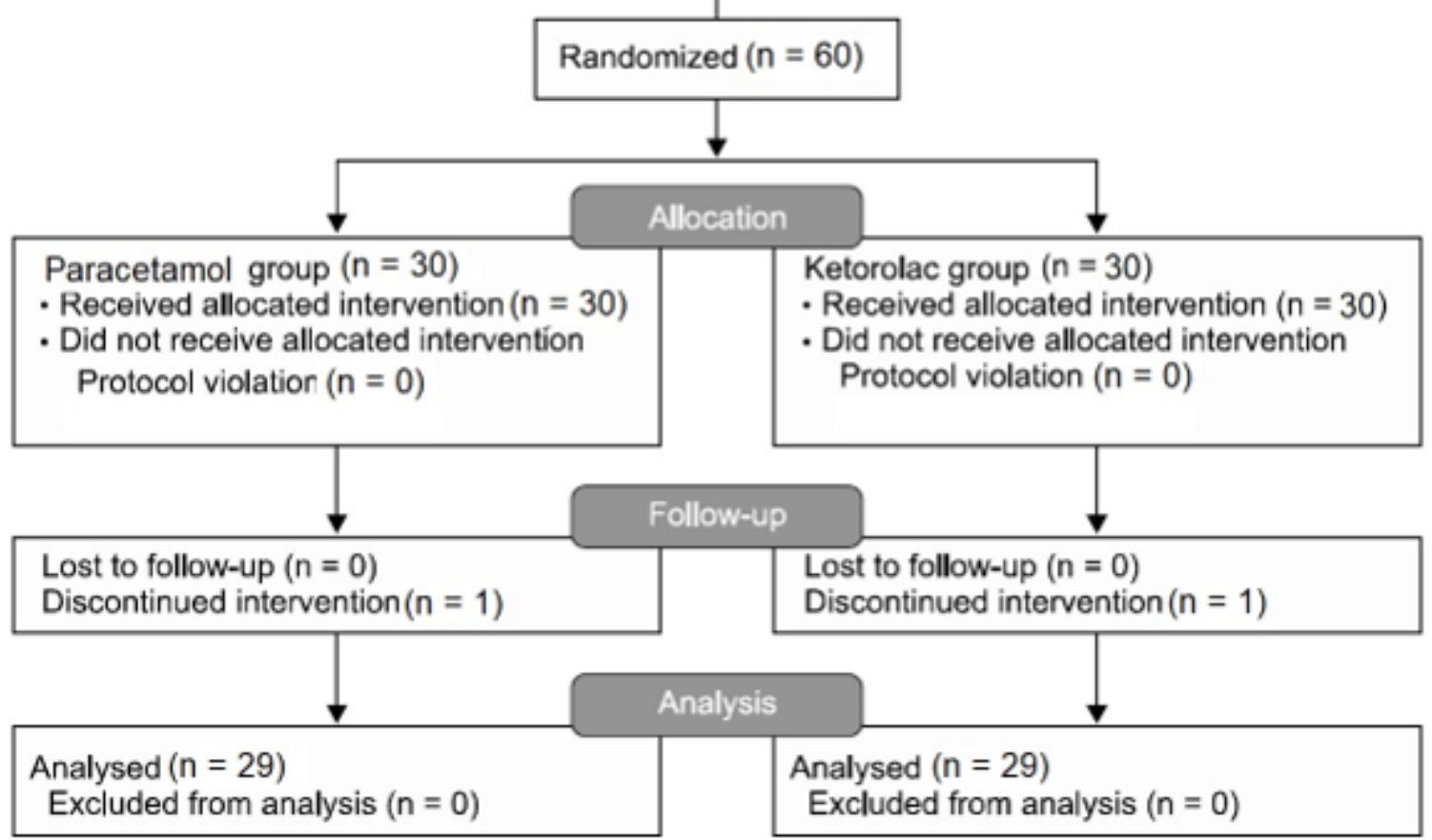

\section{Figure 1}

The consort flow chart 


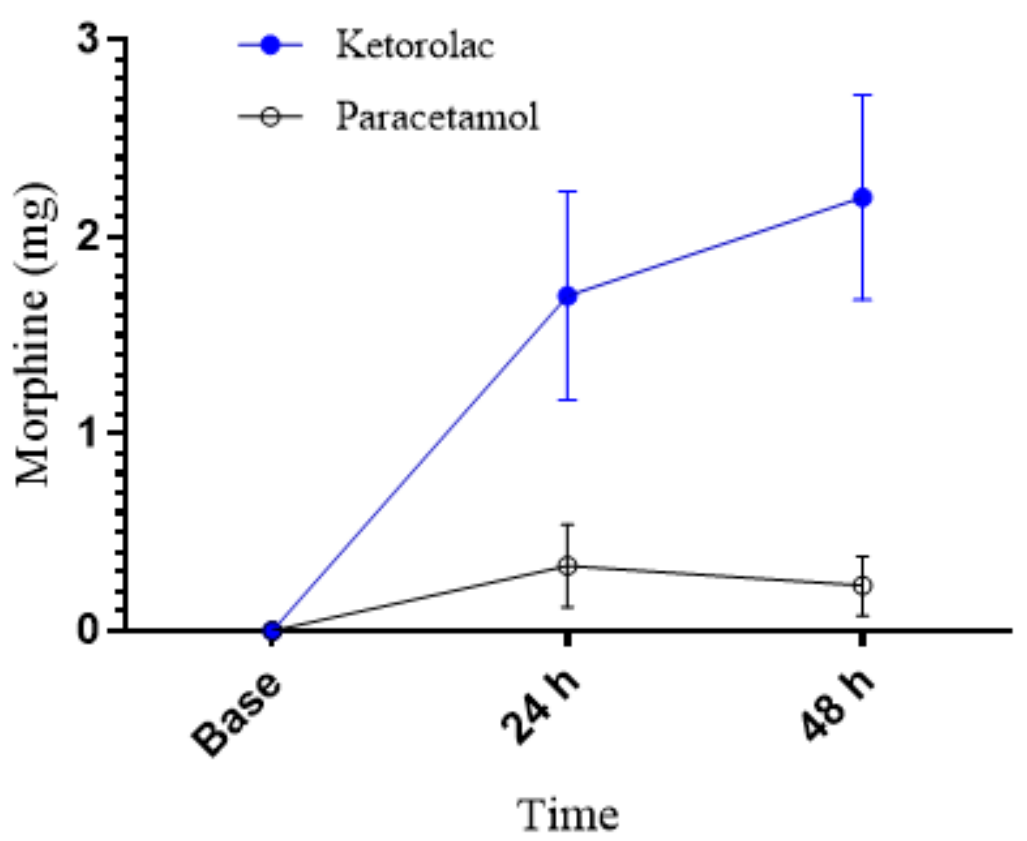

Figure 2

VAS score after the operation. There were significant VAS score declines in both groups $(P<0.05)$.

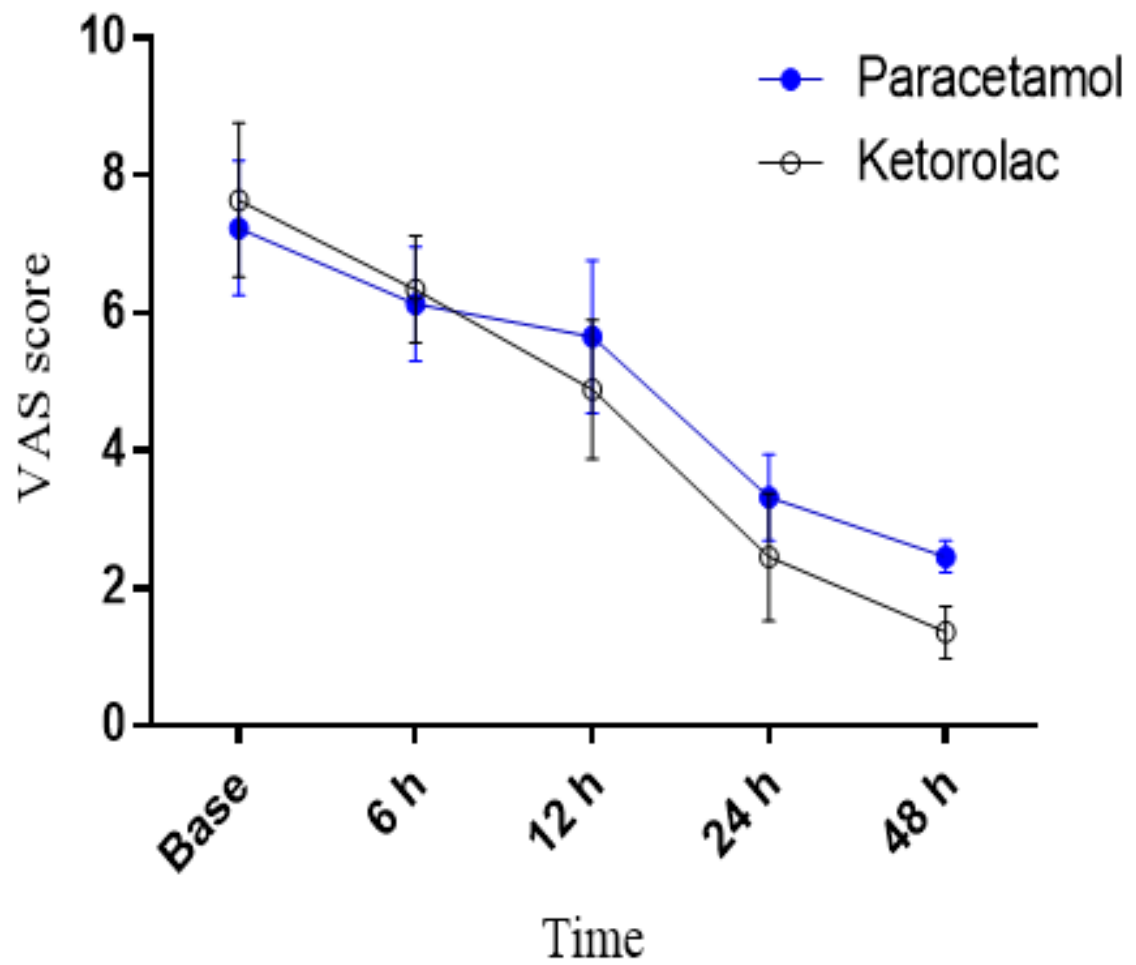

Figure 3

Postoperative morphine administration. 